\title{
Non-Markovian quantum trajectories, instruments and time-continuous measurements
}

\author{
Sven Krönke* \\ Zentrum für optische Quantentechnologien, Universität Hamburg, \\ D-22761 Hamburg, Germany \\ Walter T. Strunz ${ }^{\dagger}$ \\ Institut für Theoretische Physik, Technische Universität Dresden, \\ D-01062 Dresden, Germany
}

\begin{abstract}
The linear and the nonlinear non-Markovian quantum state diffusion equation (NMQSD) are well known tools for the description of certain non-Markovian open quantum systems. In this work, we systematically investigate whether the normalized linear NMQSD or the nonlinear NMQSD solutions can be generated by means of a time-continuous measurement. By considering any conceivable measurement scheme in the framework of instruments, we derive a necessary criterion for a measurement interpretation of both equations. Concrete examples show that the normalized linear NMQSD solutions are realizable only in the Markovian limit in general. The application of the presented criterion to the nonlinear NMQSD remains an open issue.
\end{abstract}

\section{Contents}

1 Introduction

1.1 Stochastic Schrödinger equations and open quantum systems . . . . . . . 2

1.2 Stochastic Schrödinger equations and time-continuous measurements . . . . 5

1.3 General description of time-continuous measurements . . . . . . . . . 6

2 A necessary criterion 8

2.1 Measurement outcome . . . . . . . . . . . . . . . . . 8

2.2 Stochastic evolution operators . . . . . . . . . . . 9

2.3 Scalar measures . . . . . . . . . . . . . . . . . . . . . . . . . 9

2.4 A necessary criterion . . . . . . . . . . . . . . . . . . 10

3 Application of the criterion to concrete systems 11

3.1 Jaynes-Cummings model . . . . . . . . . . . . . . . . . . . . . . . 11

3.2 Dephasing interaction . . . . . . . . . . . . . . 12

4 On the nonlinear NMQSD 13

5 Conclusions 14

*Sven.Kroenke@physnet.uni-hamburg.de

†Walter.Strunz@tu-dresden.de 


\section{Introduction}

There is no conceptual problem to assign a pure state to a classical open system like a Brownian particle. In quantum mechanics however, the entanglement between the open system and its environment complicates things. Even if the state of total quantum system is pure, the only state which can be assigned to an open quantum system is a mixture: the reduced density operator $\hat{\rho}_{\text {red }}(t)$. Obviously, the reduced density operator can be expressed as a weighted sum over pure states, which is the starting point for powerful Monte Carlo methods. As a matter of fact, this so-called unravelling is not unique [1]. On the other hand, a measurement of the environmental degrees of freedom can destroy the entanglement between the system and its environment. The open system is therefore in a well defined pure state directly after the measurement.

In order to describe the dynamics of a Markovian open quantum system by means of Monte Carlo methods, stochastic Schrödinger equations are often employed. The solutions of these evolutions equations for pure states have the remarkable property that their expectation value coincides with $\hat{\rho}_{\text {red }}(t)$. Yet such stochastic Schrödinger equations do not only occur in the context of Markovian open quantum systems but also as wave function collapse models and for the description of certain time-continuous measurements [2-6]. Moreover, there is a close relation between time-continuous measurements and the stochastic Schrödinger equations for the description of Markovian open quantum systems: The solutions of the latter evolution equations can be interpreted as trajectories of postmeasurement states conditioned on a time-continuous measurement.

In the past fifteen years, stochastic Schrödinger equations have been constructed in order to deal with general non-Markovian open quantum systems as well. Yet the understanding of these equations in the context of time-continuous measurements has not been established. Before we come to this point in more detail, some preliminaries are presented: Firstly, we review a certain stochastic Schrödinger equation approach to non-Markovian open quantum systems in section 1.1. Afterwards, the discussion so far about the relation between these equations and measurement theory is presented (section 1.2). In section 1.3. a general framework for the description of time-continuous measurements is outlined, which constitutes the basis for our systematic investigations of the interpretation of this particular stochastic Schrödinger equation in sections 2, 3 and 4 .

\subsection{Stochastic Schrödinger equations and open quantum systems}

In many settings, some system of interest, characterized by some Hamiltonian $\hat{H}$, is coupled to an environment consisting of finitely or infinitely many harmonic oscillators, where the coupling is assumed to be linear in the environmental annihilation and creation operators $\hat{a}_{\lambda}$ and $\hat{a}_{\lambda}^{\dagger}$, respectively [7]. Here the structure of the environment is determined by the oscillator frequencies $\omega_{\lambda}$ and their distribution. The coupling is characterized by the coupling strengths $g_{\lambda}$ which quantify how strongly each environmental mode $\lambda$ couples to the open system via a system coupling operator denoted by $\hat{L}$. In the interaction picture with respect to the environmental Hamiltonian $\hat{H}_{e n v}=\sum_{\lambda} \omega_{\lambda} \hat{a}_{\lambda}^{\dagger} \hat{a}_{\lambda}$, the total Hamiltonian reads

$$
\hat{H}_{\text {tot }}(t)=\hat{H} \otimes \mathbb{1}+\sum_{\lambda}\left(g_{\lambda}^{*} \hat{L} \otimes \hat{a}_{\lambda}^{\dagger} \mathrm{e}^{\mathrm{i} \omega_{\lambda} t}+g_{\lambda} \hat{L}^{\dagger} \otimes \hat{a}_{\lambda} \mathrm{e}^{-\mathrm{i} \omega_{\lambda} t}\right)
$$

for this class of open quantum systems. In the following, we consider only scenarios with the initial condition for the total system: $|\Psi(0)\rangle=\left|\psi_{0}\right\rangle \bigotimes_{\lambda}\left|0_{\lambda}\right\rangle$. Here $\left|0_{\lambda}\right\rangle$ denotes the vacuum state with respect to $\hat{a}_{\lambda}$. The environment is hence assumed to be initially at zero temperature and uncorrelated with the open system. It turns out that the embedding of the system into its environment is characterized by the environmental correlation function 
$\alpha(t-s)$ which equals

$$
\alpha(t-s)=\sum_{\lambda}\left|g_{\lambda}\right|^{2} \mathrm{e}^{-\mathrm{i} \omega_{\lambda}(t-s)}
$$

for the above initial condition. Note that this correlation function is hermitian, which means $\alpha^{*}(s-t)=\alpha(t-s)$.

If the Born-Markov approximation is applicable, $\hat{\rho}_{\text {red }}(t)$ evolves according to a master equation of the Lindblad form, where $\hat{L}$ turns out to be the corresponding Lindbladian [3.7,8]. In this paper, an open quantum system is called Markovian if and only if its reduced density operator obeys a Lindblad master equation with time-independent Lindbladians. Such a Markovian open system dynamics can only arise in the microscopic model (1) if one formally assumes $\alpha(t-s)=\kappa \delta(t-s)$. For large system Hilbert space dimensions, it is, however, more efficient to employ so-called Monte Carlo wave function techniques or quantum trajectories methods instead of solving the known operator-valued Lindblad equation numerically [2,3. These methods are based on stochastic evolution equations for pure system states as sketched above.

The dynamics of an open system becomes non-Markovian if the environment is structured and there is no time-scale separation or if the coupling is strong. Measures for the non-Markovianity of open system dynamics are currently discussed [9]. In this regime, the form of the master equation is in general unknown. Super-operator techniques are available for determining $\hat{\rho}_{\text {red }}(t)$ but often not practical [10]. Monte Carlo wave function methods, however, provide an alternative means for coping with this problem [11,12]. In this paper, we concentrate on an exact generalization of the Markovian quantum state diffusion equations to the general setting (11) which has been found by Diósi, Gisin and Strunz [13,14]. The so-called linear non-Markovian quantum state diffusion equation (linear NMQSD) reads for an initially zero temperature environment

$$
\partial_{t}\left|\psi_{t}\left(\left[z^{*}\right]_{0}^{t}\right)\right\rangle=\left(-\mathrm{i} \hat{H}+z_{t}^{*} \hat{L}-\hat{L}^{\dagger} \int_{0}^{t} \mathrm{~d} s \alpha(t-s) \frac{\delta}{\delta z_{s}^{*}}\right)\left|\psi_{t}\left(\left[z^{*}\right]_{0}^{t}\right)\right\rangle,
$$

where $\left[z^{*}\right]_{0}^{t}$ indicates a functional dependence on the drawn path of the complex Gaussian process $z_{t}^{*}$. This stochastic process is completely characterized by the moments $\mathbb{E}\left[z_{t}\right]=$ $\mathbb{E}\left[z_{t} z_{s}\right]=0$ and $\mathbb{E}\left[z_{t} z_{s}^{*}\right]=\alpha(t-s)$. By solving (3) for a deterministic initial condition $\left|\psi_{0}\right\rangle$ and many realizations of the stochastic process $z_{t}^{*}$, one can unravel the reduced density operator of the open system corresponding to $\hat{\rho}_{\text {red }}(0)=\left|\psi_{0}\right\rangle\left\langle\psi_{0}\right|$ :

$$
\hat{\rho}_{\text {red }}(t)=\mathbb{E}\left[\left|\psi_{t}\left(\left[z^{*}\right]_{0}^{t}\right)\right\rangle\left\langle\psi_{t}\left(\left[z^{*}\right]_{0}^{t}\right)\right|\right] .
$$

Here $\mathbb{E}[\cdot]$ denotes the expectation value over all solutions of (3). When reviewing the derivation of (3), one realizes that for any fixed time $t \geq 0\left|\psi_{t}\left(\left[z^{*}\right]_{0}^{t}\right)\right\rangle$ is the unnormalized relative state of the system corresponding to a coherent environmental state [13]: Let $\left|z_{\lambda}\right\rangle$ denote the unnormalized Bargmann state with $\left\langle z_{\lambda} \mid z_{\lambda}\right\rangle=\exp \left(-\left|z_{\lambda}\right|^{2}\right)$ corresponding to $\hat{a}_{\lambda}$ and let $\mathrm{d}^{2} z_{\lambda}$ be defined as $\mathrm{d} \mathfrak{R e}\left(z_{\lambda}\right) \mathrm{d} \mathfrak{I m}\left(z_{\lambda}\right)$. The state of the total system at time $t$ can then be represented as

$$
|\Psi(t)\rangle=\int \prod_{\lambda}\left(\frac{\mathrm{d}^{2} z_{\lambda} \exp \left(-\left|z_{\lambda}\right|^{2}\right)}{\pi}\right) \quad\left|\psi_{t}\left(\left[z^{*}\right]_{0}^{t}\right)\right\rangle \bigotimes_{\lambda}\left|z_{\lambda}\right\rangle
$$

if $\left[z^{*}\right]_{0}^{t}$ is given by $z_{\tau}^{*}=-\mathrm{i} \sum_{\lambda} g_{\lambda}^{*} z_{\lambda}^{*} \mathrm{e}^{\mathrm{i} \omega_{\lambda} \tau}$ where $\tau \in[0, t]$.

In the special case of a Markovian open quantum system, i.e. $\alpha(t-s)=\kappa \delta(t-s)$, the linear NMQSD reduces to the well known linear Markovian quantum state diffusion equation driven by white noise [14]. It has to be pointed out that the open system dynamics can be non-Markovian according to the definition given previously while $z_{t}^{*}$ is a Markovian stochastic process. A correlation function for which this is the case will be discussed in section 3.2 . 
The occurrence of the functional derivative in (3) is a direct consequence of the driving with coloured Gaussian noise [15]. Yet if there is a system operator $\hat{O}\left(t, s,\left[z^{*}\right]_{0}^{t}\right)$ which removes the non-locality of (3) with respect to the stochastic process, i.e.

$$
\frac{\delta}{\delta z_{s}^{*}}\left|\psi_{t}\left(\left[z^{*}\right]_{0}^{t}\right)\right\rangle=\hat{O}\left(t, s,\left[z^{*}\right]_{0}^{t}\right)\left|\psi_{t}\left(\left[z^{*}\right]_{0}^{t}\right)\right\rangle
$$

(3. 4) can be used as a Monte Carlo scheme for calculating $\hat{\rho}_{\text {red }}(t)$. If such a replacement is feasible, the linear NMQSD becomes

$$
\partial_{t}\left|\psi_{t}\left(\left[z^{*}\right]_{0}^{t}\right)\right\rangle=\left(-\mathrm{i} \hat{H}+z_{t}^{*} \hat{L}-\hat{L}^{\dagger} \int_{0}^{t} \mathrm{~d} s \alpha(t-s) \hat{O}\left(t, s,\left[z^{*}\right]_{0}^{t}\right)\right)\left|\psi_{t}\left(\left[z^{*}\right]_{0}^{t}\right)\right\rangle
$$

and one can apply parallel computing in order to solve it for many process realizations. There are many examples where the replacement (6) exists and an exact ansatz operator can be stated. Two of them will be reviewed in section 3. In other cases, approximation schemes have to be employed in order to calculate $\hat{O}\left(t, s,\left[z^{*}\right]_{0}^{t}\right)[16]$. As we will see in section 2, (77) leads to a reversible dynamics of the relative states $\left|\psi_{t}\left(\left[z^{*}\right]_{0}^{t}\right)\right\rangle$. This does not imply that $\hat{\rho}_{\text {red }}(t)$ evolves reversibly, of course. Since the right hand side of (7) is not anti-hermitian, the squared norm of a solution $\left|\psi_{t}\left(\left[z^{*}\right]_{0}^{t}\right)\right\rangle$ is not preserved under the dynamics. Yet its expectation value remains constant [14]. This observation becomes important for the following idea.

For estimating the necessary sample size for a good Monte Carlo simulation of $\hat{\rho}_{\text {red }}(t)$, it is instructive to average over normalized states $\left|\hat{\psi}_{t}\left(\left[z^{*}\right]_{0}^{t}\right)\right\rangle=\left|\psi_{t}\left(\left[z^{*}\right]_{0}^{t}\right)\right\rangle /\left\|\psi_{t}\left(\left[z^{*}\right]_{0}^{t}\right)\right\|$ in (4)). If $\nu_{0}^{t}(\cdot)$ denotes the Gaussian probability measure of the paths $\left[z^{*}\right]_{0}^{t}$, (4) can equivalently be written as

$$
\hat{\rho}_{\text {red }}(t)=\int\left|\hat{\psi}_{t}\left(\left[z^{*}\right]_{0}^{t}\right)\right\rangle\left\langle\hat{\psi}_{t}\left(\left[z^{*}\right]_{0}^{t}\right)\right| \quad\left\|\psi_{t}\left(\left[z^{*}\right]_{0}^{t}\right)\right\|^{2} \nu_{0}^{t}\left(\mathrm{~d}\left[z^{*}\right]_{0}^{t}\right)
$$

Here and from now on, we suppress a possible dependence on the complex-conjugated process realization $[z]_{0}^{t}$ in the notation 1 . According to the observations lined out above, the probability weight with which $\left|\hat{\psi}_{t}\left(\left[z^{*}\right]_{0}^{t}\right)\right\rangle$ contributes in (8), i.e. $\left\|\psi_{t}\left(\left[z^{*}\right]_{0}^{t}\right)\right\|^{2} \nu_{0}^{t}\left(\mathrm{~d}\left[z^{*}\right]_{0}^{t}\right)$, changes in the course of time. Consequently, the necessary sample size for a good estimate of $\hat{\rho}_{\text {red }}(t)$ depends on the instant $t$, which makes a simulation of the dynamics of $\hat{\rho}_{\text {red }}(t)$ difficult.

The numerical efficiency can significantly be improved by a Girsanov transformation, which results in the norm-preserving nonlinear NMQSD [14]. The latter refers to the evolution equation for the normalized states

$$
\left|\tilde{\psi}_{t}\left(\left[\tilde{z}^{*}\right]_{0}^{t}\right)\right\rangle:=\left|\hat{\psi}_{t}\left(\left[\tilde{z}^{*}\right]_{0}^{t}\right)\right\rangle
$$

where $\tilde{z}_{t}$ denotes the shifted process

$$
\tilde{z}_{t}:=z_{t}+\int_{0}^{t} \mathrm{~d} s \alpha(t-s)\left\langle\tilde{\psi}_{s}\left(\left[\tilde{z}^{*}\right]_{0}^{s}\right)|\hat{L}| \tilde{\psi}_{s}\left(\left[\tilde{z}^{*}\right]_{0}^{s}\right)\right\rangle .
$$

This process has to be regarded as a functional of the coloured noise $z_{t}$, which we omit in the notation. In this sense, the reduced density operator is also unravelled by the nonlinear NMQSD solutions:

$$
\hat{\rho}_{\text {red }}(t)=\int\left|\tilde{\psi}_{t}\left(\left[\tilde{z}^{*}\right]_{0}^{t}\right)\right\rangle\left\langle\tilde{\psi}_{t}\left(\left[\tilde{z}^{*}\right]_{0}^{t}\right)\right| \quad \nu_{0}^{t}\left(\mathrm{~d}\left[z^{*}\right]_{0}^{t}\right) .
$$

Consequently, the Girsanov transformation leads to a stochastic Schrödinger equation whose solution occur with a time-independent weight in the unravelling.

\footnotetext{
${ }^{1}$ The path probability measure $\nu_{0}^{t}$ actually depends on both $[z *]_{0}^{t}$ and $[z]_{0}^{t}$.
} 
In the following, we refer to both the normalized solutions $\left|\hat{\psi}_{t}\left(\left[z^{*}\right]_{0}^{t}\right)\right\rangle$ of the linear NMQSD and to the nonlinear NMQSD solutions $\left|\tilde{\psi}_{t}\left(\left[\tilde{z}^{*}\right]_{0}^{t}\right)\right\rangle$ as (non-Markovian) quantum trajectories. As stated above, the expectation values of those objects have a well defined physical meaning. This paper is devoted to the question whether a single quantum trajectory is physically realizable, too.

\subsection{Stochastic Schrödinger equations and time-continuous measurements}

Whether quantum trajectories are subjectively real [17, i.e. can be interpreted as trajectories of post-measurement states of a time-continuous measurement scheme, is a question motivated by both conceptual and practical interest: The variety of distinct unravellings could possibly be understood as a consequence of distinct measurement schemes [18, 19]. From an experimental point of view, a measurement scheme which generates quantum trajectories would feature a non-demolition property: If applied non-selectively upon many identical copies of the system, this scheme generates the state of the unmeasured open quantum system, i.e. $\hat{\rho}_{\text {red }}(t)$. Certainly, such a measurement scheme would depend on $\alpha(t-s)$. So one could simulate the embedding of an open system into differently structured environments.

In the Markovian regime, the interpretation of quantum trajectories is well established: Quantum state diffusion driven by real (complex) white noise can be realized by a homodyne (heterodyne) detection of the environmental photon field [3, 4]. There are many other examples in which Markovian stochastic Schödinger equations have been postulated in order to model imprecise time-continuous measurements or the dynamics of the wave function reduction (e.g. [5]). Moreover, Wiseman and Diósi have completely parameterized all diffusive stochastic Schrödinger equations for Markovian open systems and linked those equations with generating measurement schemes [19]. Some of those equations generate a non-Markovian system state dynamics while the expectation value of those states obeys a Lindblad master equation. Furthermore, the general form of a stochastic Schrödinger equation driven by independent Wiener processes has been derived such that it can be interpreted as an evolution equation for post-measurement states [20]. If one averages the post-measurement states over all measurement records, the resulting a priori state evolves according to a master equation of the Lindblad type.

However, the most general class of diffusive stochastic Schrödinger equations which allow a measurement interpretation has not been characterized in the non-Markovian regime. There are examples where such an interpretation is indeed feasible [21] and, recently, Yang, Miao and Chen proposed an interesting approach relating non-Markovian open quantum systems to time-continuous measurements [22]. In this context, see also [23]. Yet in particular, the meaning of the non-Markovian quantum trajectories is a vividly debated open question.

From (5), it becomes clear that for any time $t \geq 0$ the open system can be forced into a pure state proportional to $\left|\psi_{t}\left(\left[z^{*}\right]_{0}^{t}\right)\right\rangle$ by projectively measuring in which coherent state $\bigotimes_{\lambda}\left|z_{\lambda}\right\rangle$ the environment is. In this context, see also the work of Gambetta and Wiseman [24], who furthermore show that such a single-shot measurement interpretation leads to alternative unravellings as well. If, however, those single-shot measurements are used to constitute a time-continuous measurement scheme performed upon a single copy of the system, the system dynamics will not be given by a NMQSD because of the incompatibility of the single-shot measurements. This fact has motivated an interpretation of NMQSDs as a hidden variable theory [25. Furthermore, Diósi suggested that a certain readout schedule of initially entangled von Neumann detectors could induce the NMQSD dynamics [26]. In response, Gambetta and Wiseman showed that this scheme does not preserve the purity of the system states [27.

In [24, 25, 27], Gambetta and Wiseman argue that pure-state quantum trajectories do not exist for general non-Markovian systems within the framework of standard quantum 
mechanics. Their conclusion, however, is based only on a concrete measurement scheme which fails to generate non-Markovian quantum trajectories. In particular, the line of argument in 24 neglects the possibility of a measurement scheme which adapts conditioned on previous outcomes. Therefore, the central aim of this work is to investigate the realizability of non-Markovian quantum trajectories systematically by considering any conceivable time-continuous measurement scheme.

\subsection{General description of time-continuous measurements}

The general framework for describing quantum measurements was studied by Davies and Lewis 28, who have introduced the concept of (completely positive) instruments. In particular, this concept covers von Neumann measurements [29] but also indirect and destructive measurements (e.g. photodetection). A more recent introduction into general quantum measurements and instruments can be found in [30] and [31, respectively.

Let $\Omega$ be the set of all possible measurement outcomes and let $\mathcal{F}$ be a $\sigma$-algebra over $\Omega$ such that it contains all events which can be verified or falsified by a measurement. An event $F \in \mathcal{F}$ is called verified in an actual measurement if the measurement outcome $\omega \in \Omega$ is an element of $F$. Then a map $Y(\cdot)[\cdot]: \mathcal{F} \times \mathcal{B}(\mathbb{H}) \rightarrow \mathcal{B}(\mathbb{H})$, where $\mathcal{B}(\mathbb{H})$ denotes all bounded linear operators on the system Hilbert space $\mathbb{H}$, is called an instrument if the following conditions are fulfilled: For any $F \in \mathcal{F}, Y(F)[\cdot]$ is an operation, i.e. linear, trace-decreasing and completely positive. Besides, the normalization $\operatorname{tr}(Y(\Omega)[\hat{A}])=\operatorname{tr}(\hat{A})$ is satisfied for any $\hat{A}$. Finally, $Y(\cdot)[\hat{A}]$ is $\sigma$-additive for any $\hat{A}$.

Given the pre-measurement state $\hat{\rho}$, the probability for verifying $F \in \mathcal{F}$ is postulated to be

$$
P(F \mid \hat{\rho}):=\operatorname{tr}(Y(F)[\hat{\rho}]) \equiv \operatorname{tr}\left(\hat{\rho} Y^{\dagger}(F)[\mathbb{1}]\right),
$$

where $Y^{\dagger}(F)[\cdot]$ denotes the dual map of $Y(F)[\cdot]$. The corresponding post-measurement state is then

$$
\hat{\rho}_{F}:=\frac{Y(F)[\hat{\rho}]}{\operatorname{tr}(Y(F)[\hat{\rho}])} .
$$

A dilation theorem allows the interpretation of the stochastic dynamics (12/13) as being induced by a projective measurement upon a measurement apparatus which has been entangled with the system by some interaction 32. Such an extension is called a measuring process and is not in general unique.

A general time-continuous measurement can be described either by explicitly modelling the interplay of general single-shot measurements and short periods of free evolution (e.g. 6]) or as measurements that formally follow the mathematical framework of single-shot measurements (e.g. 20,34). Although the latter description of time-continuous measurements appears to be rather formal, it is the appropriate means to deal with the issue of this paper. The advantage of the latter description in comparison to the former is that one need not know which physical quantity has to be measured after the short periods of free evolution.

The chosen approach starts with the continuum limit: The measurement record is assumed to be a function of time instead of single numbers from the beginning. So for describing a measurement within the time interval $[0, t]$, the set of all measurement outcomes $\Omega_{0}^{t}$ needs to be an appropriate space of functions, e.g. all real- or complex-valued right continuous functions on $[0, t]$ with left limits. We denote a single record by $[x]_{0}^{t} \in \Omega_{0}^{t}$, i.e. $[x]_{0}^{t}:=(\tau \mapsto x(\tau), \tau \in[0, t])$. Naturally, the measurement record $x(\cdot)$ must be a nonanticipating stochastic process, i.e. adapted with respect to some filtration $\left\{\mathcal{F}_{t}\right\}_{t>0}$.

Then a family of instruments $\mathcal{Y}=\left\{Y_{0}^{t}(\cdot)[\cdot]: \mathcal{F}_{t} \times \mathcal{B}(\mathbb{H}) \rightarrow \mathcal{B}(\mathbb{H}) ; t>0\right\}$ describes the whole measurement. In this approach, causality has to be ensured manually: Let $F_{0}^{s} \in \mathcal{F}_{s}$ be some event which can be verified or falsified by a measurement up to time $s$. The probability for verifying $F_{0}^{s}$ must not depend on whether or not one measures longer than 
necessary, say up to time $t>s$, and then throws the additional data away. In other words, $\mathcal{Y}$ must fulfil

$$
P_{0}^{s}\left(F_{0}^{s} \mid \hat{\rho}\right)=\operatorname{tr}\left(Y_{0}^{s}\left(F_{0}^{s}\right)[\hat{\rho}]\right)=P_{0}^{t}\left(F_{0}^{s} \times \Omega_{s}^{t} \mid \hat{\rho}\right)=\operatorname{tr}\left(Y_{0}^{t}\left(F_{0}^{s} \times \Omega_{s}^{t}\right)[\hat{\rho}]\right)
$$

for any pre-measurement state $\hat{\rho}$. Here $F_{0}^{s} \times \Omega_{s}^{t}$ refers to all elements of $\Omega_{0}^{t}$ which lie in $F_{0}^{s}$ if restricted to $[0, s]$. Consequently, $\mathcal{Y}$ can only represent a time-continuous measurement scheme if the following compatibility demand is satisfied

$$
\forall 0<s<t, \quad \forall F_{0}^{s} \in \mathcal{F}_{s}: \quad\left(Y_{0}^{s}\right)^{\dagger}\left(F_{0}^{s}\right)[\mathbb{1}]=\left(Y_{0}^{t}\right)^{\dagger}\left(F_{0}^{s} \times \Omega_{s}^{t}\right)[\mathbb{1}] .
$$

According to a theorem proven by Loubenets, any instrument can be represented in a particular integral form 33 . As the realizability of certain pure post-measurement state trajectories is studied in this work, we only state the representation of instruments corresponding to efficient measurements. In this context, a measurement is called efficient if a pure pre-measurement state is turned into a pure post-measurement state as a consequence of the measurement. So if $\mathcal{Y}$ represents an efficient time-continuous measurement, there are operator-valued functions $[x]_{0}^{t} \mapsto \hat{V}_{s}^{t}\left([x]_{0}^{t}\right)$, the stochastic evolution operators, and finite scalar measures $\mu_{0}^{t}: \mathcal{F}_{t} \rightarrow I \subset[0, \infty)$ for any $0 \leq s<t$ such that

$$
Y_{0}^{t}\left(F_{0}^{t}\right)[\hat{\rho}]=\int_{F_{0}^{t}} \hat{V}_{0}^{t}\left([x]_{0}^{t}\right) \hat{\rho}\left(\hat{V}_{0}^{t}\left([x]_{0}^{t}\right)\right)^{\dagger} \quad \mu_{0}^{t}\left(\mathrm{~d}[x]_{0}^{t}\right) .
$$

The probability for finding a measurement record in the vicinity of $[x]_{0}^{t}$ reads

$$
P_{0}^{t}\left(\mathrm{~d}[x]_{0}^{t} \mid \psi\right)=\left\|\hat{V}_{0}^{t}\left([x]_{0}^{t}\right) \psi\right\|^{2} \quad \mu_{0}^{t}\left(\mathrm{~d}[x]_{0}^{t}\right)
$$

given the initial pre-measurement state $|\psi\rangle$. The corresponding post-measurement state equals

$$
\left|\psi\left([x]_{0}^{t}\right)\right\rangle=\frac{\hat{V}_{0}^{t}\left([x]_{0}^{t}\right)|\psi\rangle}{\left\|\hat{V}_{0}^{t}\left([x]_{0}^{t}\right) \psi\right\|} .
$$

By stating conditions on $\hat{V}_{s}^{t}\left([x]_{0}^{t}\right)$ and $\mu_{0}^{t}(\cdot)$ such that the compatibility condition (15) is satisfied, Barndorff-Nielsen and Loubenets have presented a general framework for the description of time-continuous measurements [34]. When applying this formalism to our problem, we start with determining the ingredients of (16) and then rederive those conditions on the ingredients from the compatibility demand (15). In particular, those conditions ensure that the instrument for an observation within $[0, t]$ can be represented as a composition of arbitrarily many instruments describing a sequence of measurements up to time $t$. Namely for any $s \in(0, t)$, one can write

$$
Y_{0}^{t}\left(\mathrm{~d}[x]_{0}^{s} \times \mathrm{d}[x]_{s}^{t}\right)[\hat{\rho}]=Y_{s}^{t}\left(\mathrm{~d}[x]_{s}^{t} \mid[x]_{0}^{s}\right)\left[Y_{0}^{s}\left(\mathrm{~d}[x]_{0}^{s}\right)[\hat{\rho}]\right],
$$

where $Y_{s}^{t}\left(\cdot \mid[x]_{0}^{s}\right)[\cdot]$ denotes an instrument which depends on the previously measured signal $[x]_{0}^{s}$ :

$$
Y_{s}^{t}\left(\mathrm{~d}[x]_{s}^{t} \mid[x]_{0}^{s}\right)[\hat{C}]:=\hat{V}_{s}^{t}\left([x]_{0}^{t}\right) \hat{C}\left(\hat{V}_{s}^{t}\left([x]_{0}^{t}\right)\right)^{\dagger} \quad \mu_{s}^{t}\left(\mathrm{~d}[x]_{s}^{t} \mid[x]_{0}^{s}\right) .
$$

Here the conditional measure $\mu_{s}^{t}\left(\mathrm{~d}[x]_{s}^{t} \mid[x]_{0}^{s}\right)=\mu_{0}^{t}\left(\mathrm{~d}[x]_{0}^{s} \times \mathrm{d}[x]_{s}^{t}\right) / \mu_{0}^{s}\left(\mathrm{~d}[x]_{0}^{s}\right)$ has been introduced. The possible dependence of $Y_{s}^{t}$ on the result $[x]_{0}^{s}$ can be seen as a generalization of instrumental processes with independent increments [31,35]: The time-continuous measurements described in [34 are allowed to adapt conditioned on previous measurement outcomes. 


\section{A necessary criterion for a measurement interpretation}

In the following, we consider only NMQSDs whose ansatz operator is noise-independent?, i.e. $\hat{O}\left(t, s,\left[z^{*}\right]_{0}^{t}\right)=\hat{O}(t, s)$ with $\hat{O}(s, s)=\hat{L}$. In order to study the transition from the nonMarkovian to the Markovian regime, we do not specify the correlation function $\alpha(t-s)$.

Suppose there is an interpretation of the normalized solution of (77) in terms of some time-continuous measurement scheme. Then there must be a family of instruments $\mathcal{Y}$ such that the following demands are satisfied:

i. These instruments are compatible with respect to time, i.e. obey (15).

ii. Given some fixed pure initial pre-measurement state, the set of all post-measurement state trajectories coincides with the set of all normalized linear NMQSD solutions corresponding to the given initial condition.

iii. If an event $F_{0}^{t} \in \mathcal{F}_{t}$ is associated with a zero probability set of normalized linear NMQSD solutions, $F_{0}^{t}$ shall be verified with zero probability in an experiment.

The minimality demand (iii) ensures that the measurement scheme generates only those normalized NMQSD solutions as post-measurement state trajectories which actually contribute to the unravelling of the reduced density operator (8). Alternatively, we could have demanded that the a priori state of the measurement scheme equals the reduced density operator. Yet such a stronger demand would blur a result which we anticipate here: Up to a certain degree, it turns out to be unimportant whether one investigates a possible measurement interpretation of the linear or of the nonlinear NMQSD. Both issues can be handled within a single framework - at least in principle.

The derivation of a necessary criterion for the measurement interpretation of nonMarkovian quantum trajectories consists of three steps: First of all, it has to be proven which quantity can be assumed to be the measurement signal w.l.o.g. (section 2.1). Secondly, the ingredients of the integral representation (16) are derived (sections 2.2 and 2.3). Finally, the compatibility demand (15) is reformulated in section 2.4. The resulting criterion can only be a necessary one since we deal with the problem on the level of instruments without referring to any experimentally realizable measuring process (cf. [31]).

\subsection{Measurement outcome}

Given some measurement signal, say $[Z]_{0}^{t}$, we must be capable of determining the corresponding trajectory of post-measurement states up to time $t$ by assumption. So we assume the existence of a map $\Phi_{t}$ from the set of all measurement records $\left\{[Z]_{0}^{t}\right\}$ to the set of all normalized linear NMQSD solutions: $\tau \mapsto\left|\hat{\psi}_{\tau}\left(\left[z^{*}\right]_{0}^{\tau}\right)\right\rangle$ with $\tau \in[0, t]$ and $\left|\hat{\psi}_{0}\left(z_{0}^{*}\right)\right\rangle=\left|\psi_{0}\right\rangle$. This map is surjective because of demand (ii). If two measurement signals $\left[Z_{1}\right]_{0}^{t},\left[Z_{2}\right]_{0}^{t}$ are associated with the same trajectory of post-measurement states, the measurement signal can be post-processed such that only $\left[Z_{1}\right]_{0}^{t}$ is displayed as the measurement outcome in both cases. Consequently, $\Phi_{t}$ is a bijection w.l.o.g.

Any process realization $\left[z^{*}\right]_{0}^{t}$ can be associated with a normalized solution of the linear NMQSD. Conversely, a given normalized linear NMQSD solution can be differentiated with respect to time. Due to the at most linear dependence of the ansatz operator on the noise, the corresponding process realization $\left[z^{*}\right]_{0}^{t}$ can iteratively be determined by comparing that time-derivative with the known equation of motion. Hence, there is a bijection, say $\Sigma_{t}$, from the set of all normalized linear NMQSD solutions within $[0, t]$ into the set of all

\footnotetext{
${ }^{2}$ The proof presented in this paper is more general: It also holds for ansatz operators which feature an additional linear functional of the noise. In the context of energy transport in molecules, a functional Taylor expansion in the noise has recently been applied in order to approximate the ansatz operator [16]. The line of argument presented here is therefore valid for systems for which this Taylor series terminates after the second term.
} 
process realizations $\left\{\left[z^{*}\right]_{0}^{t}\right\}$. As a result, $\Sigma_{t} \circ \Phi_{t}$ provides a one to one correspondence between the measurement signals $[Z]_{0}^{t}$ and the process realizations $\left[z^{*}\right]_{0}^{t}$. For this reason, $\left[z^{*}\right]_{0}^{t}$ can be assumed as the measurement signal w.l.o.g..

\subsection{Stochastic evolution operators}

Let $\hat{G}_{t}\left(\left[z^{*}\right]_{0}^{t}\right)$ be the so-called stochastic propagator which maps the initial system state $\left|\psi_{0}\right\rangle$ to the linear NMQSD solution at time $t$, i.e. $\left|\psi_{t}\left(\left[z^{*}\right]_{0}^{t}\right)\right\rangle=\hat{G}_{t}\left(\left[z^{*}\right]_{0}^{t}\right)\left|\psi_{0}\right\rangle$. It is easy to realize that the ansatz operator $\hat{O}\left(t, s,\left[z^{*}\right]_{0}^{t}\right)$ exists if and only if $\hat{G}_{t}\left(\left[z^{*}\right]_{0}^{t}\right)$ is invertible: If $\hat{G}_{t}\left(\left[z^{*}\right]_{0}^{t}\right)$ is non-singular, the ansatz

$$
\hat{O}\left(t, s,\left[z^{*}\right]_{0}^{t}\right)=\left(\frac{\delta}{\delta z_{s}^{*}} \hat{G}_{t}\left(\left[z^{*}\right]_{0}^{t}\right)\right)\left(\hat{G}_{t}\left(\left[z^{*}\right]_{0}^{t}\right)\right)^{-1}
$$

certainly satisfies (6). Conversely, if $\hat{O}\left(t, s,\left[z^{*}\right]_{0}^{t}\right)$ exists, one can integrate the evolution equation for $\hat{G}_{t}\left(\left[z^{*}\right]_{0}^{t}\right)$, i.e. effectively (17). So $\hat{G}_{t}\left(\left[z^{*}\right]_{0}^{t}\right)$ turns out to be a time-ordered exponential, which is indeed invertible. This observation allows us to define a two times propagator for any $0 \leq s \leq t$

$$
\hat{A}_{s}^{t}\left(\left[z^{*}\right]_{0}^{t}\right):=\hat{G}_{t}\left(\left[z^{*}\right]_{0}^{t}\right)\left(\hat{G}_{s}\left(\left[z^{*}\right]_{0}^{s}\right)\right)^{-1} .
$$

The two times propagator obviously obeys $\hat{A}_{s}^{t}\left(\left[z^{*}\right]_{0}^{t}\right)\left|\psi_{s}\left(\left[z^{*}\right]_{0}^{s}\right)\right\rangle=\left|\psi_{t}\left(\left[z^{*}\right]_{0}^{t}\right)\right\rangle$ and satisfies the cocycle condition

$$
\hat{A}_{s}^{t}\left(\left[z^{*}\right]_{0}^{t}\right)=\hat{A}_{r}^{t}\left(\left[z^{*}\right]_{0}^{t}\right) \hat{A}_{s}^{r}\left(\left[z^{*}\right]_{0}^{r}\right)
$$

for any $0 \leq s \leq r \leq t$. With a glance at postulate (18), we can rewrite demand (ii) equivalently as $\hat{V}_{0}^{t}\left(\left[z^{*}\right]_{0}^{t}\right) \propto \hat{A}_{0}^{t}\left(\left[z^{*}\right]_{0}^{t}\right)$. Without loss of generality, the absolute square of the proportionality factor can be absorbed in the scalar measure $\mu_{0}^{t}(\cdot)$. So up to an irrelevant phase factor, we may identify $\hat{V}_{0}^{t}\left(\left[z^{*}\right]_{0}^{t}\right)=\hat{A}_{0}^{t}\left(\left[z^{*}\right]_{0}^{t}\right)$.

\section{$2.3 \quad$ Scalar measures}

Recalling that $\nu_{0}^{t}(\cdot)$ denotes the path probability measure of the Gaussian process $\left[z^{*}\right]_{0}^{t}$, we explicate the minimality demand as follows: Any event $F_{0}^{t} \in \mathcal{F}_{t}$ with $\nu_{0}^{t}\left(F_{0}^{t}\right)=0$ is verified in a measurement up to time $t$ with the probability $P_{0}^{t}\left(F_{0}^{t} \mid \hat{\rho}\right)=0$, independently of the pre-measurement state $\hat{\rho}$. So demand (iii) translates to an absolute continuity of the operator-valued measure $\left(Y_{0}^{t}\right)^{\dagger}(\cdot)[\mathbb{1}]$ with respect to $\nu_{0}^{t}(\cdot)$ :

$$
\forall F_{0}^{t} \in \mathcal{F}_{t}: \quad \nu_{0}^{t}\left(F_{0}^{t}\right)=0 \quad \Rightarrow \quad\left(Y_{0}^{t}\right)^{\dagger}\left(F_{0}^{t}\right)[\mathbb{1}]=0 .
$$

The finite, positive scalar measure $\mu_{0}^{t}$ can be decomposed into a singular part $\mu_{\mathrm{s}}^{t}$ and an absolutely continuous part $\mu_{\mathrm{a}}^{t}$ with respect to $\nu_{0}^{t}$ by means of Lebesgue's decomposition theorem. The singular part $\mu_{\mathrm{s}}^{t}$ is concentrated on a set $C_{0}^{t} \in \mathcal{F}_{t}$ with $\nu_{0}^{t}\left(C_{0}^{t}\right)=0$ and $\mu_{\mathrm{s}}^{t}\left(\Omega_{0}^{t} \backslash C_{0}^{t}\right)=0$. Due to (24), one finds for the dual instrument

$$
\left(Y_{0}^{t}\right)^{\dagger}\left(C_{0}^{t}\right)[\mathbb{1}]=\int_{C_{0}^{t}}\left(\hat{A}_{0}^{t}\left(\left[z^{*}\right]_{0}^{t}\right)\right)^{\dagger} \hat{A}_{0}^{t}\left(\left[z^{*}\right]_{0}^{t}\right) \quad \mu_{\mathrm{s}}^{t}\left(\mathrm{~d}\left[z^{*}\right]_{0}^{t}\right)=0 .
$$

The integrand of (251) is a strictly positive definite operator as the two times propagator is invertible. Therefore, $\mu_{\mathrm{s}}^{t}\left(C_{0}^{t}\right)=0$ must hold and, consequently, $\mu_{\mathrm{s}}^{t}(\cdot)$ vanishes everywhere. According to the theorem of Radon-Nikodym, we may conclude that there is a $\nu_{0}^{t}$-a.e. unique positive functional $f_{0}^{t}\left(\left[z^{*}\right]_{0}^{t}\right)$ with $\mu_{0}^{t}\left(\mathrm{~d}\left[z^{*}\right]_{0}^{t}\right)=f_{0}^{t}\left(\left[z^{*}\right]_{0}^{t}\right) \nu_{0}^{t}\left(\mathrm{~d}\left[z^{*}\right]_{0}^{t}\right)$.

How can these functionals $f_{0}^{t}\left(\left[z^{*}\right]_{0}^{t}\right)$ be interpreted? Whereas $\hat{V}_{0}^{t}\left(\left[z^{*}\right]_{0}^{t}\right)=\hat{A}_{0}^{t}\left(\left[z^{*}\right]_{0}^{t}\right)$ ensures that the post-measurement states depend on the measurement signal in the same way as the normalized linear NMQSD solutions depend on the process realization, $f_{0}^{t}\left(\left[z^{*}\right]_{0}^{t}\right)$ determines the statistics of the measurements due to (17). Consequently, the family of 
functionals $f_{0}^{t}\left(\left[z^{*}\right]_{0}^{t}\right)$ can be seen as a means to implement the non-demolition property (8). In addition to this, $f_{0}^{t}\left(\left[z^{*}\right]_{0}^{t}\right)$ can be regarded as the Jacobian of a transformation from the actually measurement signal $[Z]_{0}^{t}$ to the process $\left[z^{*}\right]_{0}^{t}$. However, not every choice of these functionals results in a consistent family of instruments, which is the issue of the next section.

\subsection{A necessary criterion}

According to the previous sections, the normalized linear NMQSD solutions can only be generated as post-measurement states if the instruments representing the time-continuous measurement are of the following form

$$
Y_{0}^{t}\left(F_{0}^{t}\right)[\hat{\rho}]=\int_{F_{0}^{t}} \hat{A}_{0}^{t}\left(\left[z^{*}\right]_{0}^{t}\right) \hat{\rho}\left(\hat{A}_{0}^{t}\left(\left[z^{*}\right]_{0}^{t}\right)\right)^{\dagger} f_{0}^{t}\left(\left[z^{*}\right]_{0}^{t}\right) \nu_{0}^{t}\left(\mathrm{~d}\left[z^{*}\right]_{0}^{t}\right) .
$$

Each map (26) is an instrument if and only if it is normalized, i.e.

$$
\left(Y_{0}^{t}\right)^{\dagger}\left(\Omega_{0}^{t}\right)[\mathbb{1}]=\int_{\Omega_{0}^{t}}\left(\hat{A}_{0}^{t}\left(\left[z^{*}\right]_{0}^{t}\right)\right)^{\dagger} \hat{A}_{0}^{t}\left(\left[z^{*}\right]_{0}^{t}\right) \quad f_{0}^{t}\left(\left[z^{*}\right]_{0}^{t}\right) \nu_{0}^{t}\left(\mathrm{~d}\left[z^{*}\right]_{0}^{t}\right)=\mathbb{1},
$$

which is a constraint on $f_{0}^{t}\left(\left[z^{*}\right]_{0}^{t}\right)$. Moreover, the compatibility (15) has to be ensured. Let us fix $0<s<t$ and some $F_{0}^{s} \in \mathcal{F}_{s}$. Omitting all arguments, we rewrite the compatibility condition as

$$
\begin{aligned}
\mathbb{E}_{\nu_{0}^{s}}\left[\mathbf{1}_{F_{0}^{s}} f_{0}^{s}\left(\hat{A}_{0}^{s}\right)^{\dagger} \hat{A}_{0}^{s}\right] & =\mathbb{E}_{\nu_{0}^{t}}\left[\mathbf{1}_{F_{0}^{s} \times \Omega_{s}^{t}} f_{0}^{t}\left(\hat{A}_{0}^{t}\right)^{\dagger} \hat{A}_{0}^{t}\right] \\
& =\mathbb{E}_{\nu_{0}^{s}}\left[\mathbf{1}_{F_{0}^{s}} \mathbb{E}_{\nu_{s}^{t}}\left[f_{0}^{t}\left(\hat{A}_{0}^{t}\right)^{\dagger} \hat{A}_{0}^{t}\right]\right] .
\end{aligned}
$$

Here $\mathbf{1}_{A}(\cdot)$ refers to the indicator function of the set $A$ and $\mathbb{E}_{\nu_{s}^{t}}[\cdot]$ denotes the conditional expectation value given some path $\left[z^{*}\right]_{0}^{s}$. As $(28)$ holds for any $F_{0}^{s} \in \mathcal{F}_{s}$, one may conclude

$$
f_{0}^{s}\left(\hat{A}_{0}^{s}\right)^{\dagger} \hat{A}_{0}^{s}=\mathbb{E}_{\nu_{s}^{t}}\left[f_{0}^{t}\left(\hat{A}_{0}^{t}\right)^{\dagger} \hat{A}_{0}^{t}\right] \quad \nu_{0}^{s} \text {-a.e.. }
$$

Because of the cocycle property (23) and the two times propagator being invertible, the last identity may be written as

$$
\mathbb{1} f_{0}^{s}\left(\left[z^{*}\right]_{0}^{s}\right)=\int_{\Omega_{s}^{t}}\left(\hat{A}_{s}^{t}\left(\left[z^{*}\right]_{0}^{t}\right)\right)^{\dagger} \hat{A}_{s}^{t}\left(\left[z^{*}\right]_{0}^{t}\right) \quad f_{0}^{t}\left(\left[z^{*}\right]_{0}^{t}\right) \nu_{s}^{t}\left(\mathrm{~d}\left[z^{*}\right]_{s}^{t} \mid\left[z^{*}\right]_{0}^{s}\right) \quad \nu_{0}^{s} \text {-a.e. }
$$

where $\nu_{s}^{t}\left(\cdot \mid\left[z^{*}\right]_{0}^{s}\right)$ is the conditional path probability measure of $\left[z^{*}\right]_{0}^{t}$ given a path realization $\left[z^{*}\right]_{0}^{s}$. To sum up, (26) represents a time-continuous measurement scheme for every family of positive functionals $f_{0}^{t}\left(\left[z^{*}\right]_{0}^{t}\right)$ which obey the families of functional integral equations (27/30).

Now we tighten demand (iii) and require that the probability (density) to measure $\left[z^{*}\right]_{0}^{t}$ shall coincide with the probability weight of the corresponding normalized linear NMQSD solution in the unravelling (8) . This requirement necessarily leads to the choice $f_{0}^{t}\left(\left[z^{*}\right]_{0}^{t}\right) \equiv$ 1. Then the normalization (27) is fulfilled by construction [14]. In the Markovian regime $\alpha(t-s)=\kappa \delta(t-s)$, the resulting family of instruments (26) coincides with that one presented in 20]. In this limit, Barchielli and Gregoratti have proven in 20] that the compatibility is fulfilled for any Hamiltonian $\hat{H}$ and any coupling operator $\hat{L}$. 


\section{Application of the criterion to concrete systems}

In the following, we check the compatibility demand for $f_{0}^{t}\left(\left[z^{*}\right]_{0}^{t}\right) \equiv 1$ in the non-Markovian regime. Therefore, we study two simple models for which all calculations can be performed analytically without any approximations.

\subsection{Jaynes-Cummings model}

The original Jaynes-Cummings model describes the interaction of a two level atom with a single electromagnetic mode in the dipole interaction and rotating wave approximation [36]. Here we consider a generalized Jaynes-Cummings model where there is a coupling of the atom to arbitrarily many environmental modes. In particular, this model has been studied by means of NMQSDs [14. For this problem, the system Hamiltonian reads $\hat{H}=\omega / 2 \hat{\sigma}_{z}$, where $\omega$ is the atomic transition frequency and $\hat{\sigma}_{z}$ the Pauli spin matrix of the $z$-direction. The coupling operator is given by the lowering operator $\hat{L}=\hat{\sigma}_{-}$. Then the ansatz operator also turns out to be noise independent: $\hat{O}\left(t, s,\left[z^{*}\right]_{0}^{t}\right)=f(t, s) \hat{\sigma}_{-}$. Here $f(t, s)$ is a function obeying $f(s, s)=1$ and

$$
\partial_{t} f(t, s)=(\mathrm{i} \omega+F(t)) f(t, s),
$$

where $F(t):=\int_{0}^{t} \mathrm{~d} s \alpha(t-s) f(t, s)$. The corresponding linear NMQSD equals

$$
\partial_{t}\left|\psi_{t}\left(\left[z^{*}\right]_{0}^{t}\right)\right\rangle=\left(-\mathrm{i} \frac{\omega}{2} \hat{\sigma}_{z}+z_{t}^{*} \hat{\sigma}_{-}-F(t) \hat{\sigma}_{+} \hat{\sigma}_{-}\right)\left|\psi_{t}\left(\left[z^{*}\right]_{0}^{t}\right)\right\rangle .
$$

Due to the noise-independence of $\hat{O}\left(t, s,\left[z^{*}\right]_{0}^{t}\right)$, the master equation can be determined from (32) [37]:

$$
\begin{aligned}
\partial_{t} \hat{\rho}_{\text {red }}(t)= & -\mathrm{i}\left[\frac{\omega}{2} \hat{\sigma}_{z}+\mathfrak{I m}(F(t)) \hat{\sigma}_{+} \hat{\sigma}_{-}, \hat{\rho}_{\text {red }}(t)\right] \\
& +\mathfrak{R e}(F(t))\left(\left[\hat{\sigma}_{-} \hat{\rho}_{\text {red }}(t), \hat{\sigma}_{+}\right]+\left[\hat{\sigma}_{-}, \hat{\rho}_{\text {red }}(t) \hat{\sigma}_{+}\right]\right) .
\end{aligned}
$$

Consequently, the evolution equation for $\rho_{\text {red }}(t)$ is of the Lindblad form but with a timedependent Lindbladian in general. Only for $\alpha(t-s)=\kappa \delta(t-s)$, the Lindbladian becomes time-independent because one has then $F(t) \equiv \kappa / 2$. For a non-Markovian open quantum system, the decay rate $\mathfrak{R e}(F(t))$ can become negative, which does not, however, cause any problems in the NMQSD approach. In this context, see also [12].

One easily finds the two times propagator for the Jaynes-Cummings model, namely

$$
\hat{A}_{s}^{t}\left(\left[z^{*}\right]_{0}^{t}\right)=\left(\begin{array}{cc}
\mathrm{e}^{-\mathrm{i} \frac{\omega}{2}(t-s)-\int_{s}^{t} \mathrm{~d} \tau F(\tau)} & 0 \\
\mathrm{e}^{+\mathrm{i} \frac{\omega}{2}(s+t)} \int_{s}^{t} \mathrm{~d} \tau z_{\tau}^{*} \mathrm{e}^{-\mathrm{i} \omega \tau-\int_{s}^{\tau} \mathrm{d} \tau^{\prime} F\left(\tau^{\prime}\right)} & \mathrm{e}^{+\mathrm{i} \frac{\omega}{2}(t-s)}
\end{array}\right),
$$

where $(1,0)^{\mathrm{T}}$ and $(0,1)^{\mathrm{T}}$ represent the eigenvectors of $\hat{\sigma}_{z}$ corresponding to the eigenvalues +1 and -1 , respectively. Remarkably, the two times propagator depends only on the noise realization within $[s, t]$. This property has been identified by Barndorff-Nielsen and Loubenets as being one of two conditions which lead to an instrumental process with independent increments if they are simultaneously fulfilled - given that the measurement scheme is consistent, of course [34]. The integrand of the compatibility demand turns out to be of the form

$$
\left(\hat{A}_{s}^{t}\left(\left[z^{*}\right]_{0}^{t}\right)\right)^{\dagger} \hat{A}_{s}^{t}\left(\left[z^{*}\right]_{0}^{t}\right)=\left(\begin{array}{cc}
h_{s}^{t}\left(\left[z^{*}\right]_{s}^{t}\right) & j_{s}^{t}\left(\left[z^{*}\right]_{s}^{t}\right) \\
\left(j_{s}^{t}\left(\left[z^{*}\right]_{s}^{t}\right)\right)^{*} & 1
\end{array}\right) .
$$

The functionals $h_{s}^{t}(\cdot)$ and $j_{s}^{t}(\cdot)$ are stated in the appendix. According to the previous section, the following conditions must be satisfied if the normalized linear NMQSD solutions allow a measurement interpretation: $\mathbb{E}_{\nu_{s}^{t}}\left[h_{s}^{t}\right]=1$ and $\mathbb{E}_{\nu_{s}^{t}}\left[j_{s}^{t}\right]=0$ for any given 
realization $\left[z^{*}\right]_{0}^{s}$. We have explicated both conditions by discretizing the occurring path integrals and taking the left boundary points of the tiny time intervals as intermediate points. The resulting high-dimensional Gaussian integrals can be evaluated analytically. Having performed the continuum limit again, the condition $\mathbb{E}_{\nu_{s}^{t}}\left[j_{s}^{t}\right]=0$ turns into the following integral equation for the correlation function

$$
\int_{s}^{t} d \tau \alpha(\tau-u) \exp \left(\mathrm{i} \omega \tau-\int_{s}^{\tau} d \tau^{\prime} F^{*}\left(\tau^{\prime}\right)\right)=0
$$

for any $0<s<t$ and any $u \in[0, s)$. This integral equation is certainly satisfied by the correlation function of a Markovian open quantum system, i.e. $\alpha(t-s)=\kappa \delta(t-s)$. For this special case, an easy calculation verifies $\mathbb{E}_{\nu_{s}^{t}}\left[h_{s}^{t}\right]=1$. So the normalized solutions of (32) allow a measurement interpretation in the Markovian regime as expected (cf. [3,4,20]). If, however, we restrict ourselves to functions $\alpha(\cdot)$ which are Lebesgue integrable, the fundamental theorem of calculus tells us that the only solution of (36) is $\alpha(\tau)=$ 0 Lebesgue-a.e.. In this sense, the example shows that the normalized linear NMQSD solutions generically allow a measurement interpretation only in the Markovian regime.

\subsection{Dephasing interaction}

Let us consider one of the simplest open quantum systems in order to analyse whether there are certain limits in which a measurement interpretation is approximately possible. A qubit, described by $\hat{H}=\omega / 2 \hat{\sigma}_{z}$, shall be coupled to the bosonic environment in a purely dephasing way: $\hat{L}=r / \kappa \hat{\sigma}_{z} \propto \hat{H}$. Here $\kappa$ denotes some time scale and the dimensionless parameter $r$ controls the interaction strength. It is easy to show that the ansatz operator is noise-independent for this setting: $\hat{O}\left(t, s,\left[z^{*}\right]_{0}^{t}\right)=\hat{L}$ [14]. One then obtains

$$
\partial_{t}\left|\psi_{t}\left(\left[z^{*}\right]_{0}^{t}\right)\right\rangle=\left(-\mathrm{i} \frac{\omega}{2} \hat{\sigma}_{z}+z_{t}^{*} \frac{r}{\kappa} \sigma_{z}-\frac{r^{2}}{\kappa^{2}} \int_{0}^{t} \mathrm{~d} s \alpha(t-s)\right)\left|\psi_{t}\left(\left[z^{*}\right]_{0}^{t}\right)\right\rangle
$$

for the linear NMQSD, which can directly be integrated. Similarly to the previous section, the master equation can be stated and is of the Lindblad form with an in general timedependent Lindbladian:

$$
\partial_{t} \hat{\rho}_{\text {red }}(t)=-\mathrm{i}\left[\frac{\omega}{2} \hat{\sigma}_{z}, \hat{\rho}_{\text {red }}(t)\right]+\frac{r^{2}}{\kappa^{2}} \mathfrak{R e}(K(t))\left(\left[\hat{\sigma}_{z} \hat{\rho}_{\text {red }}(t), \hat{\sigma}_{z}\right]+\left[\hat{\sigma}_{z}, \hat{\rho}_{\text {red }}(t) \hat{\sigma}_{z}\right]\right),
$$

where $K(t):=\int_{0}^{t} \mathrm{~d} s \alpha(t-s)$. One finds again that the two times propagator depends only on the noise realization within $[s, t]$ :

$$
\hat{A}_{s}^{t}\left(\left[z^{*}\right]_{s}^{t}\right)=\exp \left(-\mathrm{i} \frac{\omega}{2}(t-s) \hat{\sigma}_{z}-\frac{r^{2}}{\kappa^{2}} \Theta(t, s)+\frac{r}{\kappa} \int_{s}^{t} \mathrm{~d} \tau z_{\tau}^{*} \hat{\sigma}_{z}\right)
$$

where $\Theta(t, s):=\int_{s}^{t} \mathrm{~d} \tau^{\prime} \int_{0}^{\tau^{\prime}} \mathrm{d} \tau^{\prime \prime} \alpha\left(\tau^{\prime}-\tau^{\prime \prime}\right)$. The discretized path integral of (30) with $f_{0}^{t} \equiv 1$ can be evaluated analytically. The resulting effective compatibility demand reads

$$
\forall 0<s<t, \quad \forall u \in[0, s): \quad \int_{s}^{t} d \tau \alpha(\tau-u)=0
$$

in the continuum limit. So again a measurement interpretation is exclusively possible in the Markovian regime.

It is nevertheless instructive to calculate the right hand side of the compatibility demand for the correlation function of an Ornstein-Uhlenbeck process,

$$
\alpha(t-s)=\frac{\kappa \Gamma}{2} \mathrm{e}^{-\Gamma|t-s|},
$$


which tends to $\kappa \delta(t-s)$ as $\Gamma \rightarrow \infty$. If one divides $(s, t]$ into $N$ tiny intervals of the length $\Delta t=(t-s) / N$ and defines $z_{j}^{*}:=z_{s+j \Delta t}^{*}, j \in\{0, \ldots, N\}$, the conditional path probability measure can be expressed as

$$
\begin{aligned}
\nu_{s}^{t}\left(\mathrm{~d}\left[z^{*}\right]_{s}^{t} \mid\left[z^{*}\right]_{0}^{s}\right) & =\lim _{N \rightarrow \infty} \prod_{j=1}^{N}\left(\frac{\mathrm{d}^{2} z_{j}}{\pi \kappa \Gamma^{2} \Delta t}\right) \exp \left(-\frac{\Delta t}{\kappa \Gamma^{2}} \sum_{j=1}^{N}\left|\frac{z_{j}-z_{j-1}}{\Delta t}+\Gamma z_{j-1}\right|^{2}\right) \\
& =\nu_{s}^{t}\left(\mathrm{~d}\left[z^{*}\right]_{s}^{t} \mid z_{s}^{*}\right) .
\end{aligned}
$$

With respect to this measure, the right hand side of the compatibility demand, say $\hat{C}_{R H S}\left(s, t,\left[z^{*}\right]_{0}^{s}\right)$, turns out to be a path integral with a quadratic action. Such an expression can be evaluated by employing standard techniques of path integration (e.g. [38]), which leads to

$$
\begin{aligned}
\hat{C}_{R H S}\left(s, t,\left[z^{*}\right]_{0}^{s}\right)=\exp \{ & -\frac{1}{\kappa \Gamma}\left[r^{2}\left(e^{-\Gamma t}-e^{-\Gamma s}\right)\right. \\
& \left.\left.-2 r\left(\hat{\sigma}_{z} \mathfrak{R e}\left(z_{s}\right)-r\right)\left(1-e^{-\Gamma(t-s)}\right)-\frac{r^{2}}{2}\left(1-e^{-2 \Gamma(t-s)}\right)\right]\right\} .
\end{aligned}
$$

As expected for a Markov process like the Ornstein-Uhlenbeck process, this expression depends exclusively on (the real part of) $z_{s}^{*}$ and not on the whole path $\left[z^{*}\right]_{0}^{s}$. The compatibility is clearly fulfilled in the Markovian limit:

$$
\lim _{\Gamma \rightarrow \infty} \hat{C}_{R H S}\left(s, t, z_{s}^{*}\right)=\mathbb{1} .
$$

Trivially, the compatibility holds in the limit of vanishing interaction $r \rightarrow 0$. The system dynamics then becomes unitary and independent of the measurement signal $\left[z^{*}\right]_{0}^{t}$, which means that the dynamics is induced by a trivial measurement. Moreover, the compatibility can be achieved approximately either in the weak coupling and short correlation time limit $\kappa \Gamma \gg 1$, which is the physical Markov limit in fact, or in the short-time limit $(t-s) \ll 1 / \Gamma$ and $(t-s) \ll \kappa$. In the latter scenario, the correlation function is approximately constant on the considered time scales, $\alpha(t-s) \approx \kappa \Gamma / 2$, which results in linear dependence of $z_{t}$ on $z_{s}^{*}$ almost surely, namely $z_{t} \approx z_{s}^{*} \approx 0$. By inspecting the two times propagator (39), one can easily show that the system dynamics turns out to be effectively unitary: The considered times are much smaller than the time scale of the interaction. Therefore, the system may be regarded as being decoupled from its environment. Finally by inspecting (43), one realizes that there is no meaningful long-time limit such as $(t-s) \gg 1 / \Gamma$ which approximately leads to compatibility.

For a fair discussion of these results, one has to pay respect to the involved time scales. Since the measurement is described by a family of instruments $\mathcal{Y}$, the continuum limit is anticipated. Consequently, the time span between two consecutive measurements, which constitute the time-continuous measurement scheme, is arbitrarily short and thus the shortest involved time scale - unless the open system is Markovian. In the latter case, the time span between two consecutive measurements is comparable with the time scale of the environmental dynamics.

\section{On the nonlinear NMQSD}

In this section, we show that the necessary criterion (26/27/30) can also be used for investigating a possible measurement interpretation of the nonlinear NMQSD in principle.

Due to the linear dependence of the ansatz operator on the noise and the shift relation (10), there is a bijection between the process realizations $\left[z^{*}\right]_{0}^{t}$ and solutions of the nonlinear NMQSD up to time $t$. So $\left[z^{*}\right]_{0}^{t}$ can be assumed as the measurement signal w.l.o.g.. Moreover, (9) indicates that $\left|\tilde{\psi}_{t}\left(\left[\tilde{z}^{*}\right]_{0}^{t}\right)\right\rangle$ depends on the realization $\left[\tilde{z}^{*}\right]_{0}^{t}$ in the 
same way as $\left|\hat{\psi}_{t}\left(\left[z^{*}\right]_{0}^{t}\right)\right\rangle$ depends on $\left[z^{*}\right]_{0}^{t}$. Therefore, we may conclude $\hat{V}_{0}^{t}(\cdot)=\hat{A}_{0}^{t}(\cdot)$ with a glance at (18). So the normalized linear NMQSD solutions can be turned into nonlinear NMQSD solutions by appropriately weighting the driving process realizations $\left[z^{*}\right]_{0}^{t}$. When reviewing the derivation of the nonlinear NMQSD [14, one realizes that the path probability measure of the shifted process $\tilde{z}_{t}^{*}$ is absolutely continuous with respect to the path probability measure of $z_{t}^{*}$, i.e. $\nu_{0}^{t}(\cdot)$. Consequently, the minimality demand (iii) implies $\mu_{0}^{t}\left(\mathrm{~d}\left[z^{*}\right]_{0}^{t}\right)=f_{0}^{t}\left(\left[z^{*}\right]_{0}^{t}\right) \nu_{0}^{t}\left(\mathrm{~d}\left[z^{*}\right]_{0}^{t}\right)$ again. As a result, any family of instruments which might generate the nonlinear NMQSD solutions as trajectories of post-measurement states must be of the form (26).

So if the nonlinear NMQSD can be interpreted in terms of a time-continuous measurement scheme, there must be a family of functionals $f_{0}^{t}\left(\left[z^{*}\right]_{0}^{t}\right)$ such that (27) and (30) hold and that the pure post-measurement state trajectories occur with the same probability (density) as they appear as nonlinear NMQSD solutions.

\section{Conclusions}

In this paper, we have considered NMQSDs whose ansatz operators depend at most linearly on the stochastic process $z_{t}^{*}$. For this class of open quantum systems, we have derived a necessary criterion for the measurement interpretation of non-Markovian quantum trajectories. By employing a representation theorem for instruments, any time-continuous measurement scheme which is conceivable in the framework of standard quantum mechanics has been taken into account. In particular, this criterion covers both "direct" and "indirect" measurements. Here the attribute "direct" refers to general measurements which are implemented by some measurement apparatus in the sense of the dilation theorem (cf. [32]). In contrast to this, "indirect" measurements bear on settings where the given harmonic environment acts as a quantum probe (e.g. heterodyne detection). By tracing out the environmental degrees of freedom, any instrument on the bipartite Hilbert space of the "indirect" measurement setting reduces to an instrument acting on the system Hilbert space as long as the initial state is not entangled. Hence, the above formalism also applies in this case.

A word of caution is in order here: The proof presented in this paper is based on the assumption that any measurement can be described by a completely positive instrument. Yet an entangled initial state of the total system could lead to indirect measurements upon the system which cannot be described by complete positive maps (cf. [39]).

We have especially concentrated on the interpretation of the linear NMQSD: For a suitable choice of $f_{0}^{t}$, the family of instruments (26) generates the normalized solutions of the linear NMQSD. In the Markovian regime, the resulting measurement scheme coincides with the family of instruments presented in [20], where the compatibility of these instruments is shown for any finite dimensional system.

By investigating examples, we have shown that in general the normalized solutions $\left|\hat{\psi}_{t}\left(\left[z^{*}\right]_{0}^{t}\right)\right\rangle$ of the linear NMQSD allow a measurement interpretation only if $\alpha(t-s)=$ $\kappa \delta(t-s)$. The compatibility of these measurements is violated in the non-Markovian regime. As our analysis is performed on the level of instruments without considering a concrete measuring processes, a descriptive explanation for this failure cannot be given. We may only conclude that in general causality is violated by any measurement scheme which might generate the normalized linear NMQSD solutions. At least, one can state certain limits in which the compatibility is approximately satisfied.

Up to now, it remains an open question whether the nonlinear NMQSD solutions allow a measurement interpretation in the non-Markovian regime. We hope that (26]27|30) can explicitly be applied to some system in order to investigate this question systematically. Our investigation moreover shows a mathematical peculiarity of the unravelling (8) in comparison to (11) in the non-Markovian regime: In section 3, we have effectively proven 
that $\left\|\psi_{t}\left(\left[z^{*}\right]_{0}^{t}\right)\right\|^{2}$ is not in general a $\left(\mathcal{F}_{t}\right)$-martingale if $\alpha(t-s) \neq \kappa \delta(t-s)$. This implies that the family of probability measures $\left\{\int .\left\|\psi_{t}\left(\left[z^{*}\right]_{0}^{t}\right)\right\|^{2} \nu_{0}^{t}\left(\mathrm{~d}\left[z^{*}\right]_{0}^{t}\right), t>0\right\}$ is not consistent. Consequently, one can numerically perform a Monte Carlo simulation of $\hat{\rho}_{\text {red }}(t)$ based on the normalized linear NMQSD solutions $\left|\hat{\psi}_{t}\left(\left[z^{*}\right]_{0}^{t}\right)\right\rangle$ but there is no overall path probability measure for the driving process $z_{t}^{*}$ in this case. The Girsanov transformation, however, shifts this inconsistency of the measures into the dynamics of the nonlinear NMQSD solutions. The effectively driving process $z_{t}^{*}$ is then Gaussian and one can show that Kolmogorovs extension theorem is applicable for any $\alpha(t-s)$. This difference in the unravellings does not occur for the Markovian quantum state diffusion as $\left\|\psi_{t}\left(\left[z^{*}\right]_{0}^{t}\right)\right\|^{2}$ turns out to be a $\left(\mathcal{F}_{t}\right)$-martingale (cf. [20]).

It would be interesting to invert the question discussed in this paper. Namely, is there a family of functionals $f_{0}^{t}$ such that the functional integral equations (27/30) are satisfied and the a priori state $Y_{0}^{t}\left(\Omega_{0}^{t}\right)\left[\left|\psi_{0}\right\rangle\left\langle\psi_{0}\right|\right]$ coincides with $\hat{\rho}_{\text {red }}(t)$ ?

Finally, it is important to notice that the proof of (26/27/30) mainly bases on measuretheoretical arguments. Therefore, the criterion (27/30) can be applied to a broader class of stochastic Schrödinger equations, which is defined by the following two demands: First, any of these stochastic Schrödinger equations is either linear or can be formulated in a linear version. This linearity implies that the corresponding stochastic propagator is invertible. Second, this linear equation must be linearly driven by some stochastic process. For any of these stochastic Schrödinger equations, (26]2730) provides a tool to investigate whether the randomness of its solutions possibly originates from a time-continuous measurement.

\section{Acknowledgement}

We would like to thank Elena R. Loubenets for the enlightening discussion about the quantum stochastic representation of time-continuous measurements and Philip M. R. Brydon for his helpful comments on the manuscript. S.K. thanks Benjamin TrendelkampSchroer for their inspiring discussions.

\section{Appendix}

For the Jaynes-Cummings model, the operator-valued integrand of (30) contains the following two functionals

$$
\begin{aligned}
h_{s}^{t}\left(\left[z^{*}\right]_{s}^{t}\right):= & \exp \left[-2 \int_{s}^{t} \mathrm{~d} \tau \mathfrak{R e}(F(\tau))\right]+\int_{s}^{t} \mathrm{~d} \tau_{1} \int_{s}^{t} \mathrm{~d} \tau_{2} z_{\tau_{1}} z_{\tau_{2}}^{*} \quad \times \\
& \times \quad \exp \left[\mathrm{i} \omega\left(\tau_{1}-\tau_{2}\right)-\int_{s}^{\tau_{1}} \mathrm{~d} \tau_{1}^{\prime} F^{*}\left(\tau_{1}^{\prime}\right)-\int_{s}^{\tau_{2}} \mathrm{~d} \tau_{2}^{\prime} F\left(\tau_{2}^{\prime}\right)\right], \\
j_{s}^{t}\left(\left[z^{*}\right]_{s}^{t}\right):= & \int_{s}^{t} \mathrm{~d} \tau z_{\tau} \exp \left[\mathrm{i} \omega(\tau-s)-\int_{s}^{\tau} \mathrm{d} \tau^{\prime} F^{*}\left(\tau^{\prime}\right)\right] .
\end{aligned}
$$

\section{References}

[1] Hughston L. P., Jozsa R. and Wootters W. K. (1993). A complete classification of quantum ensembles having a given density matrix. Phys. Lett. A 183, 14-8.

[2] Gisin N. and Percival I. C. (1992). The quantum-state diffusion model applied to open systems. J. Phys. A: Math. Gen. 25, 5677-91.

Dalibard J., Castin Y. and Mølmer K. (1992). Wave-function approach to dissipative processes in quantum optics. Phys. Rev. Lett. 68, 580-3. 
Gardiner C. W., Parkins A. S. and Zoller P. (1992). Wave-function quantum stochastic differential equations and quantum-jump simulation methods. Phys. Rev. A 46, 436381.

Percival I. C. (1998). Quantum State Diffusion (Cambridge: Cambridge University Press).

[3] Carmichael H. (1993). An Open Systems Approach to Quantum Optics (Lecture Notes in Physics. Monographs vol 18, Berlin: Springer-Verlag).

[4] Milburn G. J. and Wiseman H. M. (1993). Quantum theory of field-quadrature measurements. Phys. Rev. A 47, 642-62.

Milburn G. J. and Wiseman H. M. (1993). Interpretation of quantum jump and diffusion processes illustrated on the Bloch sphere. Phys. Rev. A 47, 1652-66.

[5] Diósi L. (1988). Continuous quantum measurement and Itô formalism. Phys. Lett. A 129, 419-23.

Diósi L. (1988). Quantum stochastic processes as models for state vector reduction. J. Phys. A: Math. Gen. 21, 2885-98.

Belavkin V. P. (1989). A new wave equation for a continuous nondemolition measurement. Phys. Lett. A 140, 355-358.

Belavkin V. P. (1989). A continuous counting observation and posterior quantum dynamics. J. Phys. A: Math. Gen. 22, L1109-14.

Ghirardi G. C., Pearle P. and Rimini A. (1990). Markov processes in Hilbert space and continuous spontaneous localization of systems of identical particles. Phys. Rev. A 42, 78-89.

Belavkin V. P. and Staszewski P. (1992). Nondemolition observation of a free quantum particle. Phys. Rev. A 45, 1347-56.

[6] Barchielli A, and Belavkin V. P. (1991). Measurements continuous in time and a posteriori states in quantum mechanics. J. Phys. A: Math. Gen. 24, 1495-514.

[7] Breuer H.-P. and Petruccione F. (2002). The Theory of Open Quantum Systems (Oxford: Oxford University Press).

Weiss U. (2008). Quantum Dissipative Systems (Series in Modern Condensed Matter Physics vol 13, 3rd edition, Singapore: World Scientific).

[8] Lindblad G. (1976). On the generators of quantum dynamical semigroups. Commun. math. Phys. 48, 119-30.

[9] Breuer H.-P., Laine E.-M. and Piilo J. (2009). Measure for the degree of nonMarkovian behavior of quantum processes in open systems. Phys. Rev. Lett. 103, 210401-4.

Huelga S. F., Rivas Á. and Plenio M. B. (2010). Entanglement and non-Markovianity of quantum evolutions. Phys. Rev. Lett. 105, 50403-6.

[10] Nakajima S. (1958). On quantum theory of transport phenomena. Prog. Theor. Phys. 20, 948-59.

Zwanzig R. (1960). Ensemble method in the theory of irreversibility. J. Chem. Phys. 33, 1338-41. 
[11] Grabert H. and Stockburger J. T. (2001). Non-Markovian quantum state diffusion. Chemical Physics 268, 249-256.

Breuer H.-P. (2004). Genuine quantum trajectories for non-Markovian processes. Phys. Rev. A 70, 12106-17.

[12] Härkönen K., Maniscalco S., Suominen K.-A. and Piilo J. (2008). Non-Markovian quantum jumps. Phys. Rev. Lett. 100, 180402-5.

[13] Diósi L. and Strunz W. T. (1997). The non-Markovian stochastic Schrödinger equation for open systems. Phys. Lett. A 235, 569-73.

[14] Gisin N., Diósi L. and Strunz W. T. (1998). Non-Markovian quantum state diffusion. Phys. Rev. A 58, 1699-1712.

[15] Budini A. A. (2000). Non-Markovian Gaussian dissipative stochastic wave vector. Phys. Rev. A 63, 12106-16.

[16] Diósi L., Gisin N., Strunz W. T. and Yu T. (1999). Non-Markovian quantum-state diffusion: perturbation approach. Phys. Rev. A 60, 91-103.

Eisfeld A., Roden J., Strunz W. T. and Wolff W. (2009). Influence of complex excitonphonon coupling on optical absorption and energy transfer of quantum aggregates. Phys. Rev. Lett. 103, 58301-4.

Eisfeld A., Roden J. and Strunz W. T. (2011). Non-Markovian quantum state diffusion for absorption spectra of molecular aggregates. J. Chem. Phys. 134, 34902-18.

[17] Wiseman H. M. (1996). Quantum trajectories and quantum measurement theory. Quantum Semiclass. Opt. 8, 205-22.

[18] Vaccaro J. A. and Wiseman H. M. (1998). Maximally robust unravelings of quantum master equations. Phys. Lett. A 250, 241-248.

[19] Diósi L. and Wiseman H. M. (2001). Complete parameterization, and invariance, of diffusive quantum trajectories for Markovian open systems. Chemical Physics 268, 91-104.

Diósi L. and Wiseman H. M. (2001). Erratum to "Complete parameterization, and invariance, of diffusive quantum trajectories for Markovian open systems". Chemical Physics 271, 227.

[20] Barchielli A. and Gregoratti M. (2009). Quantum Trajectories and Measurements in Continuous Time. The Diffusive Case (Lecture Notes in Physics vol 782, Berlin: Springer-Verlag).

[21] Barchielli A., Pellegrini C. and Petruccione F. (2010). Stochastic Schrödinger equations with coloured noise. Europhysics Letters 91, 24001.

[22] Yang H., Miao H. and Chen Y. (2011). Non-adiabatic elimination of auxiliary modes in continuous quantum measurements. Preprint arXiv:1108.0963v3.

[23] Diósi L. (2011). Non-Markovian open quantum systems: input-output field, memory, monitoring. Preprint arXiv:1108.3763v1.

[24] Gambetta J. and Wiseman H. M. (2002). Non-Markovian stochastic Schrödinger equations: generalization to real-valued noise using quantum-measurement theory. Phys. Rev. A 66, 12108-24. 
[25] Gambetta J. and Wiseman H. M. (2003). Interpretation of non-Markovian stochastic Schrödinger equations as a hidden-variable theory. Phys. Rev. A 68, 62104-12.

[26] Diósi L. (1990). Relativistic theory for continuous measurement of quantum fields. Phys. Rev. A 42, 5086-92.

Diósi L. (2008). Non-Markovian continuous quantum measurement of retarded observables. Phys. Rev. Lett. 100, 80401-4.

Diósi L. (2008). Erratum to "Non-Markovian continuous quantum measurement of retarded observables". Phys. Rev. Lett. 101, 149902.

[27] Gambetta J. and Wiseman H. M. (2008). Pure-state quantum trajectories for general non-Markovian systems do not exist. Phys. Rev. A 101, 140401-4.

[28] Davies E. B. and Lewis J. T. (1970). An operational approach to quantum probability. Commun. Math. Phys. 17, 239-60.

[29] Lüders G. (1951). Über die Zustandsänderung durch den Meßprozeß. Ann. Phys., Lpz. 8, 322-328.

Von Neumann J. (1955). Mathematical Foundations of Quantum Mechanics (Princeton, NJ: Princeton University Press).

[30] Milburn G. J. and Wiseman H. M. (2009). Quantum Measurement and Control (Cambridge: Cambridge University Press).

[31] Holevo A. S. (2001). Statistical Structure of Quantum Theory (Lecture Notes in Physics. Monographs vol 67, Berlin: Springer-Verlag).

[32] Ozawa M. (1984). Quantum measuring processes of continuous observables. J. Math. Phys. 25, 79-87.

[33] Loubenets E. R. (2001). Quantum stochastic approach to the description of quantum measurements. J. Phys. A: Math. Gen. 34, 7639-75.

[34] Barndorff-Nielsen O. E. and Loubenets E. R. (2002). General framework for the behaviour of continuously observed open quantum systems. J. Phys. A: Math. Gen. 35, $565-88$.

[35] Barchielli A., Lanz L. and Prosperi G. (1983). Statistics of continuous trajectories in quantum mechanics: operation-valued stochastic processes. Found. Phys. 13, 779812.

Barchielli A. and Holevo A. S. (1995). Constructing quantum measurement processes via classical stochastic calculus. Stochastic Process. Appl. 58, 293-317.

Barchielli A., Paganoni A. M. and Zucca F. (1998). On stochastic differential equations and semigroups of probability operators in quantum probability. Stochastic Process. Appl. 73, 69-86.

[36] Cummings F. W. and Jaynes E. T. (1963). Comparison of quantum and semiclassical radiation theories with application to the beam maser. Proceedings of the IEEE 51, 89-109.

[37] Strunz W. T. and Yu T. (2004). Convolutionless non-Markovian master equations and quantum trajectories: Brownian motion. Phys. Rev. A 69, 52115-28. 
[38] Schulman L. S. (1981). Techniques and Applications of Path Integration (New York: Wiley).

[39] Pechukas P. (1994). Reduced dynamics need not be completely positive. Phys. Rev. Lett. 73, 1060-2. 